\title{
Orientation and Rotational Motions of Single Molecules by Polarized Total Internal Reflection Fluorescence Microscopy (polTIRFM)
}

\author{
John F. Beausang, Yujie Sun, Margot E. Quinlan, Joseph N. Forkey, and Yale E. Goldman
}

In this article, we describe methods to detect the spatial orientation and rotational dynamics of single molecules using polarized total internal reflection fluorescence microscopy (polTIRFM). polTIRFM determines the three-dimensional angular orientation and the extent of wobble of a fluorescent probe bound to the macromolecule of interest. We discuss single-molecule versus ensemble measurements, as well as single-molecule techniques for orientation and rotation, and fluorescent probes for orientation studies. Using calmodulin (CaM) as an example of a target protein, we describe a method for labeling CaM with bifunctional rhodamine (BR). We also describe the physical principles and experimental setup of polTIRFM. We conclude with a brief introduction to assays using polTIRFM to assess the interaction of actin and myosin.

The goal of most single-molecule experiments is to measure the structural, mechanical, or biochemical dynamics of an individual molecule while it interacts with partner ligands or performs a specific function. In this article, we focus on detecting the orientation and rotational motions of molecules using physical signals produced by extrinsic, polarized fluorescent probes. The physical signals that emanate from these molecules carry information about orientation and rotational motions and can be used successfully to study structural dynamics.

Rotations are essential to the functions of energy-transducing enzymes, molecular motors, nucleicacid-processing enzymes, and many other types of cellular machines. For example, the helical structures of nucleic acids and chromatin imply that untwisting motions are essential during processes such as DNA replication and transcription. Enzymes such as topoisomerases, helicases, transcription activators, and histone modifiers have evolved to process nucleic acids and handle the requisite rotational motions necessary to untwist, untie, unfold, package, and repair DNA (Pollard and Earnshaw 2002; Seidel and Dekker 2007). In the mitochondrial $\mathrm{F}_{1} \mathrm{~F}_{\mathrm{o}}$ ATP synthase, the $\mathrm{F}_{\mathrm{o}}$ subunit converts a transmembrane proton gradient into rotations of a shaft connecting to the $\mathrm{F}_{1}$ subunit, which phosphorylates ADP to produce three ATP molecules per rotation (Senior et al. 2002). Crystal structures of the macromolecular pumps that produce transmembrane ionic gradients for use in energy storage, cotransport, and signaling have revealed large tilting motions that are essential to the pumping mechanism (Toyoshima and Inesi 2004). The light-chain domain of myosin acts as a rotating lever arm that magnifies subnanometer structural changes at the ATP-binding site into nanometer-scale motions to propel cargo along the axis of actin (Goldman 1998; Geeves and Holmes 2005). The angular position

Adapted from Single-Molecule Techniques (ed. Selvin and Ha). CSHL Press, Cold Spring Harbor, NY, USA, 2008.

(C) 2012 Cold Spring Harbor Laboratory Press

Cite this article as Cold Spring Harb Protoc; 2012; doi:10.1101/pdb.top069344 
J.F. Beausang et al.

and stress-strain mechanics of the lever arm also sense the load and feed back to control the biochemical steps of the motor's ATPase cycle. Thus, detecting the orientation and rotational motions of subdomains of these macromolecules is crucial for understanding their mechanisms.

In this article, we explain how to measure single-molecule orientation and rotational motions using polTIRFM. We discuss the advantages and limitations of polTIRFM, and we compare polTIRFM to other single-molecule techniques as well as to bulk experiments that supply rotational information on ensembles of molecules.

\section{SINGLE-MOLECULE VERSUS ENSEMBLE MEASUREMENTS}

Why do experimentalists struggle with the tiny, noisy signals obtained from individual molecules? The signals obtained from classical biochemical and biophysical ensemble experiments are strong and relatively free of noise because they are averages among a large population of molecules (usually $>>$ $\left.10^{10}\right)$. A substantial disadvantage of ensemble experiments, however, is that the biophysical measurements-when obtained by averaging-discard some information about the differences among members of the population. Although this variation may not be important, some of the discarded information might be pertinent to understanding the details of the underlying mechanism. The principal advantage of single-molecule experiments is that the detailed reaction trajectory of each individual molecule is monitored directly, which eliminates the blurring caused by averaging.

In a population of molecules, the lack of temporal synchrony, thermal fluctuations, and true inhomogeneity among individual molecules can lead to distributions of their properties. The breadth of this distribution may be characterized in detail; as in structural nuclear magnetic resonance (NMR) spectroscopy (Igumenova et al. 2006), it may be temporally halted by freezing (Costello 2006), or it may be nearly eliminated by forcing each molecule into the same configuration in a crystal (Chiu et al. 2006). But, for all of these experiments, averaging over the entire ensemble is still required. A general technique for reducing temporal variation between molecules is to synchronize them by initiating a reaction abruptly (e.g., a stop-flow experiment) or by perturbing their equilibrium (e.g., a temperature jump) (Gutfreund 1995). The degree of synchronization among members of the ensemble, however, is lost within a few reaction steps because each enzymatic or conformational transition takes place stochastically. This quickly decaying synchronization makes detecting reversals, pauses, and long reaction trajectories (e.g., transcription and translation) very difficult. Thermal fluctuations of the macromolecule are often mechanistically crucial-such as in the diffusive search of myosin V for its next actin-binding site (Veigel et al. 2002)—but are typically lost by averaging.

For orientation and rotational motions, a suspension or solution of molecules is usually distributed isotropically in space, so that no static directional information is available. The extent and rate of rotational wobble among members of the group and between domains within a molecule can be determined by applying a perturbation, such as a brief polarized laser pulse. Polarization refers to the direction of the oscillating electric field and can be used to preferentially excite those molecules that are aligned along the electric field. Such photoselection is similar to partially synchronizing the rotational motions of the molecules in the population; the photoselected subgroup of molecules is oriented within a specific distribution of angles relative to the polarized excitation pulse. This anisotropy decays with the rotational correlation time of the molecules, and the decay is faster when segmental internal rotations of subdomains are present (Lakowicz 2006). The excited state lifetime (most commonly a few nanoseconds for fluorescence and microseconds for phosphorescence) restricts the observable rates of rotational motions to these timescales.

Some biological samples are exceptions to the usual isotropic nature of ensembles and have directional order imposed on their structural components. Examples include muscle fibers, lipid bilayer membranes, and multilayer lipid lamellae. In these cases, a symmetry axis termed "the director" is a reference axis for comparing molecular orientation. The axial angle is readily detectable in the ordered ensemble and is very pertinent to the mechanism; for instance, the tilting of the myosin lever 
arm relative to a muscle fiber axis is the structural cause of force production (Irving et al. 1995; Geeves and Holmes 2005). However, azimuthal angular information around the director, which may also be mechanistically relevant, is not available.

The single-molecule approach usually eliminates the need to synchronize members of a distribution because the trajectory of each member is measured separately from that of the other molecules. As a result, fluctuations of structure, reversals, pauses, reaction dwell times, forces, and displacements are detected directly. When an averaged property is useful, it can be constructed by summing the individual single-molecule recordings. These advantages come at the expense of limited signal-to-noise properties of the instrumentation and/or limited time resolution. With noisy signals, distinguishing truly brief state occupancies and rare events from variations caused by instrumental and sampling statistics requires careful analysis (Min et al. 2005; Shaevitz et al. 2005).

In contrast to molecular assemblies that operate together, some molecules work by themselves or in very small groups. Orienting and synchronizing them during activity may not be possible or may even disturb their function. The myosin $\mathrm{V}$ molecular motor is an example of a biological machine that operates nearly on its own and is not synchronized with its neighbors. In such cases, detecting functionally relevant rotational motions is not feasible in a bulk suspension but requires tracking of single molecules. Thus, methods for determining the orientation and rotational motions from single molecules provide complementary, or sometimes essential, mechanistic information that is difficult or impractical to obtain using classical ensemble techniques.

\section{SINGLE-MOLECULE TECHNIQUES FOR ORIENTATION AND ROTATION}

Signals that carry angular information from intrinsic components of macromolecules, such as infrared absorption of carbonyl bonds and fluorescence of tryptophan residues, are not strong enough to be detected individually with the available technology. Thus, in single-molecule orientational studies, a tag or marker is added to the domain of interest. Small polymer beads and fluorescent cytoskeletal filaments have been used as effective markers because they are readily observed by optical microscopy (Kinosita 1999; Ali et al. 2002; Hua et al. 2002; Gore et al. 2006). Beads offer the advantage that they can be rotationally manipulated in optical and magnetic traps (Strick et al. 2000; La Porta and Wang 2004). These markers are generally large relative to the labeled domain, however, and can substantially influence the dynamics of the domain (Kinosita et al. 2004; Shiroguchi and Kinosita 2007).

In contrast, fluorescent probes are small molecules that can be introduced readily into engineered sites on proteins and nucleic acids. Although it is still crucial to test the functional integrity and dynamics of the labeled target macromolecule, a large number of successful examples have been described recently (Marks and Nolan 2006; Waggoner 2006). Experiments designed to evaluate the position and distribution of such labeled proteins and nucleic acids have become a mainstay of cell biology. Hyper-resolution microscopy techniques have evolved, providing information about the localization of individual fluorophores at nanometer-scale precision (Yildiz et al. 2003) and forming images with better than 50-nm resolution (Hell et al. 2004; Betzig et al. 2006; Rust et al. 2006).

In the present article, we focus on determining the spatial orientation and rotations of fluorescently labeled molecules using polTIRFM. In polTIRFM, the angular dependence of the probe's absorption and emission is used to determine its three-dimensional orientation by comparing the relative intensity of the fluorescence emission at known excitation and detection polarizations. Other single-molecule fluorescence polarization techniques directly image a two-dimensional projection of the radiation pattern of the fluorescent molecule (Sick et al. 2000; Bartko et al. 2002; Kreiter et al. 2002; Lieb et al. 2004). Defocused orientational and positional imaging (DOPI) is one example, where the microscope is intentionally defocused slightly to emphasize features of the radiation distribution pattern that indicate the probe's orientation. DOPI enables relatively straightforward determination of the full hemisphere of dipole orientations with useful angular and temporal resolution. Compared with polTIRFM, the instrumentation in DOPI is simpler to implement and can simultaneously determine the probe's spatial position, a feature that has only been partially implemented with 
J.F. Beausang et al.

polTIRFM (Syed et al. 2006). The temporal resolution of DOPI $(\sim 0.5-1 \mathrm{sec})$ is lower than for polTIRFM ( $\sim 40-80 \mathrm{msec})$, but in many cases, the biological reaction can be slowed down-for instance, by reducing the substrate concentration - to match the time resolution of the method (Toprak et al. 2006). The angular resolutions of the two techniques are comparable $\left(\sim 10-20^{\circ}\right)$. polTIRFM, however, is sensitive to the rate and amplitude of the rotational wobbling motions of the probe (and the labeled macromolecule), whereas DOPI and similar techniques provide the average angle over the recording gate time.

\section{FLUORESCENT PROBES FOR ORIENTATION STUDIES}

Fluorescent probes often approximate electromagnetic dipoles that preferentially absorb and emit light polarized along their dipole axis. With xanthene-derivative fluorophores such as rhodamine and cyanine-derivative fluorophores such as $\mathrm{Cy} 3$, the visible absorption and emission dipole moments are coaligned with the long axis of the molecule (Chen and Bowman 1965; Penzkofer and Wiedmann 1980). This property simplifies the data analysis and increases the accessible orientational information relative to fluorophores that do not display coaligned absorption and emission dipole moments (van der Heide et al. 1992), because the azimuthal motions of the probe around its own axis are inconsequential. Probes that have a known and stable local orientation relative to the structure of the target molecule are especially informative because the orientation of their optical dipoles corresponds directly to the rotations of interest. Several types of labeling strategies have been described with probes containing two or four linker groups, providing fixed local orientation between the two attachments (Corrie et al. 1999; Adams et al. 2002). In other cases, the local orientation of a probe can be estimated by computer modeling (Sale et al. 2002) or by X-ray crystallography (Otterbein et al. 2001).

Bifunctional rhodamine (BR; Corrie et al. 1998), which possesses two iodoacetamide linkers flanking the chromophore, has been used to cross-link cysteine (Cys or C) residues in the myosin regulatory light chain (Corrie et al. 1999; Hopkins et al. 2002), muscle troponin-C (Ferguson et al. 2003; Sun et al. 2006), and calmodulin (CaM). Figure 1 (top left) shows the BR-CaM that is prepared in Fluorescent Labeling of Calmodulin with Bifunctional Rhodamine (Beausang et al. 2012a) and is used in Fluorescent Labeling of Myosin V for Polarized Total Internal Reflection Fluorescence Microscopy (polTIRFM) Motility Assays (Beausang et al. 2012b). Two Cys residues are placed seven residues apart in an $\alpha$-helix (at positions 66 and 73) of the CaM sequence. In this way, the side chains of the cysteine residues face nearly the same direction, two turns apart, and can be efficiently cross-linked by the bifunctional reactive probe. Although there is some wobble (halfcone angle, $25^{\circ}$ ), the orientation of the probe's dipole is aligned between the two Cys $\beta$-carbon atoms (Mercier et al. 2003). Several mutants with other pairs of inserted Cys residues provide probes with different orientations that are also known relative to the structure of the target molecule. The two residues do not need to be contained within the same helix (Hopkins et al. 2002; Brack et al. 2004). A commercial version of $B R$, with slightly more rigid linker groups, is available (bis- $[(N-$ iodoacetyl)piperazinyl]sulfonerhodamine; Invitrogen). This compound has been shown to crosslink Cys residues in kinesin (Sosa et al. 2001; Asenjo et al. 2006) and in TnC (M. Irving, pers. comm.), possibly with a more restricted range of spacing between the two linked amino acids (Sosa et al. 2001).

The method described in Fluorescent Labeling of Calmodulin with Bifunctional Rhodamine (Beausang et al. 2012a) for labeling CaM is based on the concept that maintaining a very low concentration of both peptide and free bifunctional probe during the labeling reaction improves the yield of properly cross-linked product relative to undesired species, such as two fluorophores bound to one protein, two proteins bound to one fluorophore, and higher order conjugates. Thus, small amounts of probe are added successively to the reaction mixture. After the labeling reaction is quenched with sodium 2-mercaptoethanesulfonate (MESNA), the mixture is purified on reversephase high-performance liquid chromatography (HPLC) with an acetonitrile gradient and refolded by 

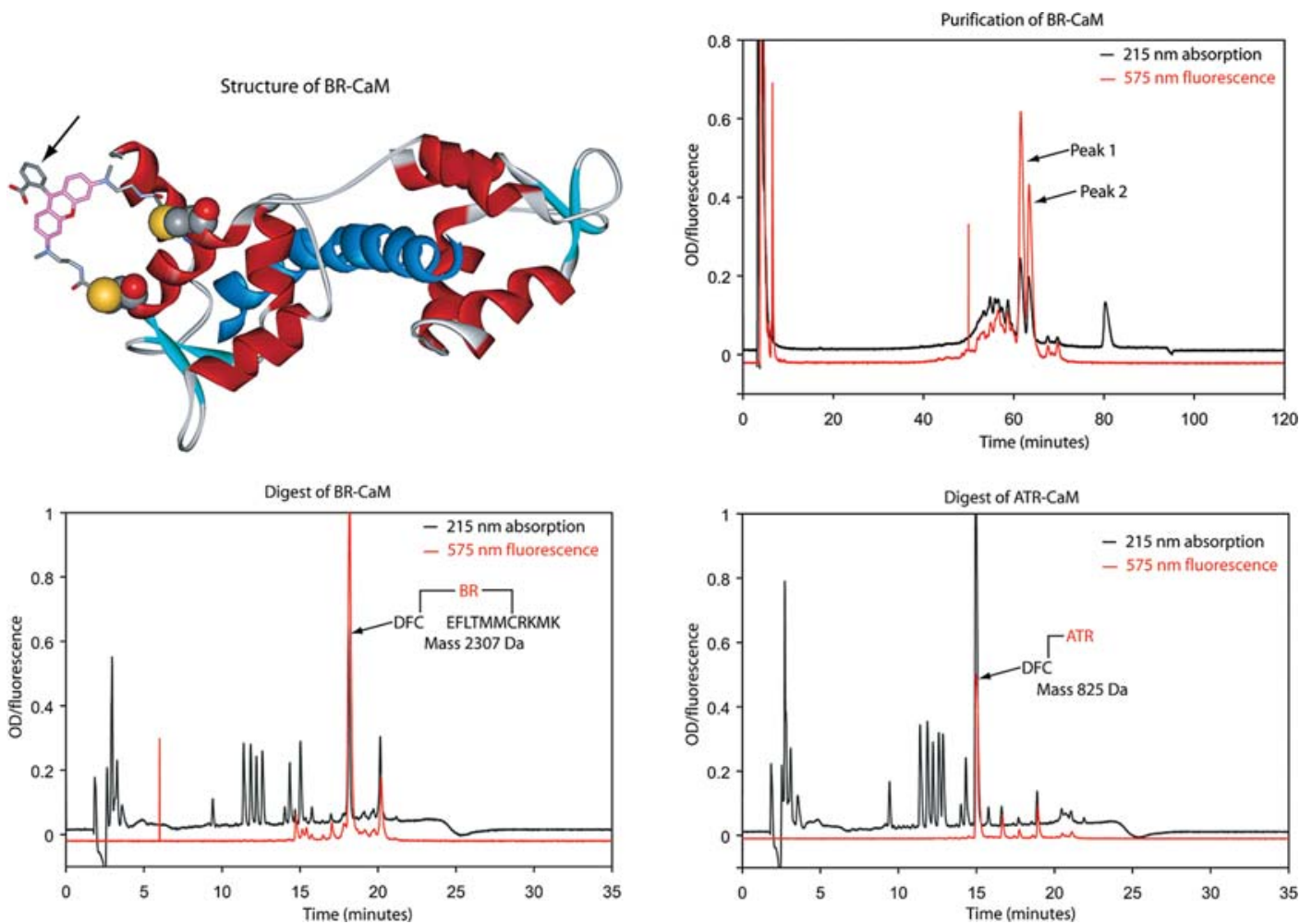

FIGURE 1. Purification and characterization of bifunctional rhodamine-calmodulin (BR-CaM). (Top left) The crystal structure of CaM (Houdusse et al. 1996) showing the location and orientation of the BR entity. The arrow identifies the carboxyphenyl ring of BR that produces the two diastereoisomer peaks appearing in the figure at the top right. Reversephase (C18) HPLC of labeled CaM (top right) and tryptic fragments (bottom left/right).

dialysis into an exchange buffer. The HPLC recording in Figure 1 (top right) shows two fluorescently labeled peaks, each of which has one fluorophore per protein chain and a total mass of 17,266 daltons. We presume these two peaks to be two diastereoisomers of the same adduct having the pendant carboxyphenyl ring (Fig. 1, top left) facing opposite directions. These isomers have no appreciable functional differences (Hopkins et al. 2002). Purified and refolded BR-CaM maintains its function, as evidenced by a gel-shift assay (Burgess et al. 1980), and does not affect motility when exchanged into myosin $\mathrm{V}$ and myosin VI.

To confirm that the two Cys residues in the labeled CaM are actually cross-linked, a sample of the purified fluorescent protein is digested by an endoproteinase that cleaves the peptide adjacent to the labeling site and in between the two linker Cys residues. In the case of the BR-CaM discussed here, a portion of the relevant amino acid sequence is GTIDFCEFLTMMCRKMKDTD, where the two Cys residues positioned for $\mathrm{BR}$ attachment are underlined. Endoproteinase Asp- $\mathrm{N}$ cleaves peptides on the amino-terminal side of aspartic acid (Asp or D) residues, and under prolonged incubation conditions, it also cleaves amino-terminal to glutamic acid (Glu or E). Thus, proteolysis produces fragments DFC and EFLTMMCRKMK, but in BR-linked CaM, these two peptides are covalently linked through the fluorophore, giving the expected mass of peptide plus probe equal to $2307 \mathrm{Da}$, as shown in Figure 1 (bottom left). A control experiment with 5-iodoacetamidotetramethylrhodamine (5-IATR, a monofunctional fluorophore), labeling the single-Cys mutant GTIDFCEFLTMMARKMKDTD, produced the fragment DFC-ATR, with the expected mass of 825 Daltons (Fig. 1, bottom right). Several other bifunctionally labeled proteins have been similarly analyzed by mass spectroscopy of proteolytic fragments (Corrie et al. 1999; Sosa et al. 2001; Ferguson et al. 2003).

Verifying the purification of cross-linked end products and functional testing of these labeled proteins are essential aspects of polTIRFM experiments. An independent analytical method uses gel shifts after reaction with maleimide and thiol derivatives of polyethylene glycol to assay for free thiols 
J.F. Beausang et al.

and free iodoacetamides (Kosolapov and Deutsch 2003). This technique has been used mostly to detect folding and oligomerization of nascent channel proteins (Robinson et al. 2006), but it also correctly distinguishes two-site-labeled BR-CaM from other products of the BR-labeling reaction.

\section{THE PRINCIPLES OF poITIRFM}

The evanescent field generated in TIRFM provides the low fluorescent background necessary for detecting single molecules. Its polarization is directly controlled by the incident illumination, allowing for robust excitation of the probe with polarizations oriented along all three orthogonal spatial directions. The production of light polarized along the optical axis of a conventional or confocal microscope is possible but more complicated (Wilson et al. 2003). The orientation of the probe optical dipole relative to the polarization of the excitation light strongly affects the amount of light absorbed and thus emitted. The detected intensity is similarly affected by the relative orientation between the probe and the analyzer. These two effects can be used to infer the three-dimensional orientation of the probe by exciting it sequentially with light of several known polarizations and simultaneously detecting the fluorescence emission at multiple polarizations. In addition to the static spatial orientation, the polarized fluorescence signals provide dynamic information about the mobility of the probe on a nanosecond timescale and, separately, information about the extent of protein motions on a microsecond timescale. These motions are caused by the rotations of the probe and protein; they are represented by a fast wobble cone with half-angle $\delta_{\mathrm{f}}$ and nanosecond rotational correlation time $\tau_{\mathrm{f}}$ and by a slow wobble cone with half-angle $\delta_{s}$ and microsecond correlation time $\tau_{s}$. The theory and practical implementation of these additional signals have been described earlier (Ha et al. 1998; Forkey et al. 2000, 2003; Rosenberg et al. 2005), but the two timescales for wobble motions have not yet been fully exploited for biological experiments at the single-molecule level.

For the polTIRFM setup we discuss here, all of the fluorescent photons that are collected are used for determining orientational information, implying that the probe's precise spatial position is not determined at the same time. This limitation is not fundamental, and the simultaneous detection of position and orientation is possible (Enderline et al. 2005; Syed et al. 2006). The total number of parameters determined in polTIRFM is five: $\theta, \phi, \delta_{\mathrm{s}}, \delta_{\mathrm{f}}$, and $\kappa$. The parameters $\theta$ and $\phi$ are the axial and azimuthal angles, respectively, of the probe dipole, where, in a coordinate system, the $z$ axis points along the optical axis of the microscope and the $x$ and $y$ axes lie in the plane of the microscope stage (Fig. 2). $\mathrm{K}$ is the intensity of the total collected emission from the probe.

The photon absorption/detection probabilities are proportional to the cosine-squared of the angle between the excitation/detection polarizers and the dipole axis. This property and the nanosecond and microsecond rotational dynamics (wobble) can be combined into a model that predicts the measured polarized fluorescent intensities from $\theta, \phi, \delta_{s}, \delta_{\mathrm{f}}$, and $\kappa$. polTIRFM thus discriminates spatial orientation with sensitivity related to the fourth power of the cosine of these angles. It is important to realize that a dipole is not a vector pointing in a unique direction, but rather a symmetric rod pointing in two directions: $(\theta, \phi)$ and $\left(180^{\circ}-\theta, 180^{\circ}+\phi\right)$. This symmetry is intrinsic to an oscillating dipole and
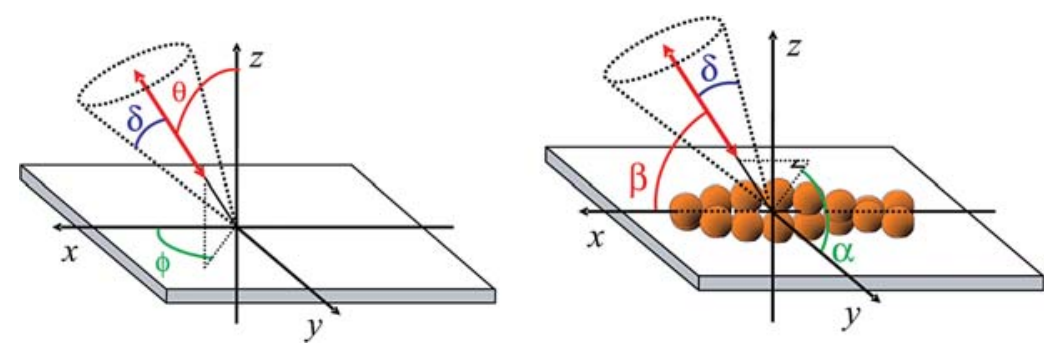

FIGURE 2. Angular coordinates in (left) the microscope reference frame and (right) the actin-filament reference frame, where the double-headed arrow (red) represents the dipole of the fluorescent probe. 
electromagnetic radiation and reduces the range of detection to a hemisphere. Depending on the geometry of the experimental setup, additional symmetries may exist among various probe angles (e.g., $\phi$ and $-\phi$ ) that can further reduce the ability to discriminate the final angular position and obscure the interpretation of the data. These geometrical symmetries can be resolved using multiple excitation/detection paths (including multiple polarizations within a path).

In our setup, the fluorescent emission is collected by a microscope objective, and projected through a polarizing beam splitter onto two photon counters that resolve the $x$ and $y$ polarizations from the image plane (Fig. 3). The result of a typical experiment is a group of 8-16 measured intensities corresponding to a complete set of excitation and detection polarization combinations from which one value of $\left(\theta, \phi, \delta_{s}, \delta_{\mathrm{f}}, \kappa\right)$ is determined. Time courses of $\left(\theta, \phi, \delta_{\mathrm{s}}, \delta_{\mathrm{f}}, \kappa\right)$ can be obtained by collecting data for repeated cycles of the excitation polarizations.

At laser intensities that allow 5-10-sec recording time before irreversible photobleaching of BR or tetramethylrhodamine, sufficient emitted photons $(\sim 50-100)$ are registered in $5-10 \mathrm{msec}$ to estimate $\theta$ and $\phi$ with $5-15^{\circ}$ uncertainty. Thus, for eight input and two detector polarizations that enable the discrimination of orientation in a full hemisphere, the time resolution is $80 \mathrm{msec}$. The time-multiplexed nature of this technique mandates that photophysical flickering (blinking) of the probe fluorescence be minimized while still maintaining protection of the probe from rapid irreversible photobleaching. We have found for rhodamine and Cy3 that 50-100 mM dithiothreitol (DTT) in the sample buffer is an effective antifade reagent and virtually eliminates the blinking observed with enzymatic deoxygenating systems (Quinlan et al. 2005; Rosenberg et al. 2005). Another redox compound, Trolox, has also been reported to serve as an effective antibleach additive that does not produce blinking (Rasnik et al. 2006).

Numerical techniques are required to determine $\left(\theta, \phi, \delta_{\mathrm{s}}, \delta_{\mathrm{f}}, \kappa\right)$ from polTIRFM intensities because the probe orientations are nonlinearly related to the intensities, which have relatively large fluctuations caused by the statistics of counting the low numbers of photons. We implement a Levenberg-Marquardt solver, programmed in C, to find the combination of $\left(\theta, \phi, \delta_{s}, \delta_{\mathrm{f}}, \kappa\right)$ that maximizes the likelihood that the intensities calculated from the probe model match the measured intensities (Press et al. 1992). The necessary equations are listed in Forkey et al. (2005).

After determining $\left(\theta, \phi, \delta_{s}, \delta_{f}, \kappa\right)$ for each complete cycle of measured intensities, angular ambiguities caused by the geometrical symmetries of the experimental setup must be resolved. The
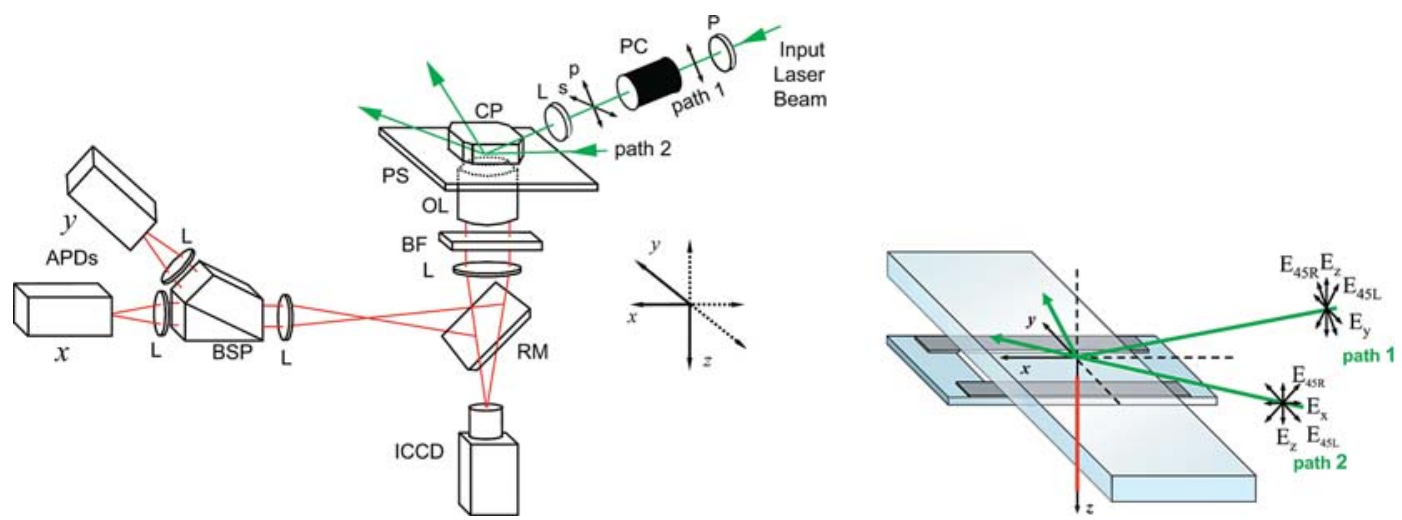

FIGURE 3. The poITIRFM experimental apparatus. (Left) The input laser beam (green) is alternately directed along paths 1 and 2. For each path, the beam passes through a linear polarizer $(\mathrm{P})$ and then a Pockels cell (PC) to generate alternating linear polarizations parallel $(p)$ and perpendicular $(s)$ to the relevant reflection plane. The beam is coupled into the quartz microscope slide by passing through the coupling prism (CP) and through index-matching liquid. At the quartz/water interface, total internal reflection occurs, sending the beam back out through the coupling prism to a beam dump (not shown). The quartz slide (top left) is mounted on a piezoelectric stage (PS). Fluorescence (red) is collected by a microscope objective lens (OL), passed through bandpass filters (BF), and imaged by lenses (L). Depending on the position of a removable mirror (RM), the fluorescence is imaged either onto an intensified CCD (ICCD) camera or through a Thompson beam-splitting prism (BSP) onto two avalanche photodiodes (APDs). (Right) Orientation of the flow cell relative to the two paths. 
J.F. Beausang et al.

selection of a reasonable biologically or physiologically relevant frame of reference, instead of the microscope coordinate frame, facilitates the interpretation of the data. For instance, in the study of motor proteins, the cytoskeletal track along which motion occurs (e.g., actin) is a natural reference frame. $\theta$ and $\phi$ are converted to $\beta$, the axial angle of the probe relative to the actin filament, here oriented along the $+x$ axis in the microscope frame, and $\alpha$, the azimuthal angle measured from the $+y$ axis toward the $+z$ axis (Fig. 2). Three different representations of the probe's orientation vector $\mathrm{O}$ facilitate this coordinate transformation:

$$
\underbrace{\left(\begin{array}{c}
O_{x} \\
O_{y} \\
O_{z}
\end{array}\right) \Leftrightarrow\left(\begin{array}{c}
\sin (\theta) \cos (\phi) \\
\sin (\theta) \sin (\phi) \\
\cos (\theta)
\end{array}\right)}_{\begin{array}{c}
\text { Microscope } \\
\text { Reference Frame }
\end{array}} \Leftrightarrow\left(\begin{array}{c}
\cos (\beta) \\
\sin (\beta) \cos (\alpha) \\
\sin (\beta) \sin (\alpha)
\end{array}\right)
$$

The transforms $\beta=\cos ^{-1}(\sin (\theta) \cos (\phi)), \alpha=\arctan 2(\cos (\theta)$, and $\sin (\theta) \sin (\phi))$ follow directly. Note that $\arctan 2(x, y)$ is similar to the standard $\arctan (y / x)$ function but single-valued over a larger range $\left(-180^{\circ}<\alpha<180^{\circ}\right.$ for $\arctan 2$ and $-90^{\circ}<\alpha<90^{\circ}$ for $\left.\arctan \right)$. In making this transformation, a particular hemisphere must be chosen for the scope of $\beta$ and $\alpha$. As an example, for molecular motors walking along actin fixed to a microscope slide (in the processive motility assay), the hemisphere on the aqueous side of the glass/water interface is a reasonable choice. For an actin filament translocating along myosin attached to the slide and sparsely labeled with a fluorescent dye (in the twirling assay), a hemisphere centered on the filament axis (front or rear) is the correct choice.

A polTIRFM setup consists of three optical systems: excitation, collection, and detection. The excitation system includes the laser (which emits the appropriate wavelength to excite the fluorophore), Pockels cells (to dynamically alter the polarization), waveplates, and mirrors (to direct the beam onto the slide/water interface at the required glancing angle for total internal reflection). For BR or tetramethylrhodamine, either 532- or 514-nm laser light works well because the laser intensity can easily achieve usable excitation rates (with limited photobleaching), and excitation on the shortwavelength side of the absorption peak at $550 \mathrm{~nm}$ minimizes leakage of scattered excitation light through the longer wavelength emission filters.

Note that mirrors, especially high-reflectance dielectric mirrors consisting of multiple thin film layers, can introduce phase shifts and thus ellipticity upon reflection, possibly corrupting the desired polarization. These phase shifts are introduced when the desired polarization is intermediate between the polarizations that are perpendicular $(s)$ and parallel $(p)$ to the plane containing the incident and reflected beams (the scattering plane). A Berek variable waveplate (New Focus, Inc.) is a stable mechanical phase compensator with adjustable rotational position and birefringence that converts the elliptically polarized radiation produced by the Pockels cell and mirrors into a beam with arbitrary linear polarization set by the voltages on the Pockels cells. We adjust the voltages and compensator settings by measuring extinction through a crossed linear polarizer placed in each beam just before the prism or objective that couples it to the sample interface.

We describe here the main features of a prism-type polTIRFM setup (Fig. 3); further details can be found in Forkey et al. (2005). Two orthogonal beams are directed alternately onto the sample through a BK-7 glass prism at a glancing angle to generate the evanescent field at the interface between a quartz slide (index of refraction, $n=1.46)$ and the aqueous sample $(n=1.33)$. The microscope slide lies in the $x-y$ plane on a computer-controlled piezoelectric stage with the excitation from beams 1 and 2 propagating in the $x-z$ and $y-z$ planes, respectively. Each beam is linearly polarized in two directions: $s$ and $p$. Beams 1 and 2 generate evanescent waves that are predominantly polarized in the $y$ and $z$ and 
the $x$ and $z$ directions, respectively. For the $p$ polarized beams, the evanescent fields are slightly elliptical because of a phase shift of the electric field component parallel to the sample plane (e.g., see Forkey et al. 2000). The analysis software accounts for this small component, but for simplicity, we refer to the major component in the $z$ direction.

Switching between the two beam paths is achieved by a Pockels cell that alternates the laser polarization between vertical and horizontal every $20 \mathrm{msec}$ and by a polarizing beam splitter. This design is susceptible to interference fringes at the sample if extinction between the two paths is not sufficient. We achieve a 1:1000 extinction ratio between the two paths with a feedback circuit that corrects for any thermal drift in the Pockels cell (Forkey et al. 2005). The polarization of each beam is again switched back and forth between horizontal and vertical every $10 \mathrm{msec}$ by another Pockels cell in each path. The beam is focused onto the sample plane by a relatively long focal length lens $(100 \mathrm{~mm})$, illuminating a region $\sim 50 \mu \mathrm{m} \times 100 \mu \mathrm{m}$ at the sample. The result of switching the paths and polarizations is a 40 -msec cycle of four input polarizations: $s 1, p 1, s 2$, and $p 2$ (corresponding to polarizations predominantly in the $y, z, x$, and $z$ directions, respectively).

The optics used for efficiently collecting the single-molecule fluorescence emission are contained within a conventional epiillumination light microscope, equipped with a $100 \times, 1.2$ numerical aperture (NA) water-immersion objective lens. A significant fraction of the light collected by such a high numerical lens is refracted through a large angle, which mixes otherwise orthogonal polarizations and requires correction during the analysis (Axelrod 1989). In our setup, we minimize the impact of the high autofluorescence naturally found in glass by passing the excitation light through the low autofluorescence quartz slide and imaging through a relatively thin glass coverslip (Fig. 4). In addition, the thick quartz slide, combined with the large excitation beam angles, ensures that autofluorescence from the glass prism is outside of the field of view of the objective. Fluorescent emission is passed through two filters (Chroma; HQ575/90M and HQ545LP), which act as a bandpass filter to block scattered laser light from being detected. Fluorescence is then directed by a movable mirror either onto an intensified charge-coupled device (ICCD) camera for general imaging or through a polarizing beam-splitting prism onto the avalanche photodiodes (APDs) for detection of the $x$-and $y$-polarized emission.

Typically, a field of sparsely distributed fluorophores is imaged via the camera; a single fluorophore is selected manually by mouse-clicking on the image display monitor; the fluorophore is centered above the APDs using software that drives the piezoelectric stage; and then the movable mirror is positioned to direct the emission onto the two APDs for recording the polTIRFM signals. Two output polarizations for each input polarization result in eight intensities per cycle: ${ }_{{ }_{1} 1} I_{x, s_{1} 1} I_{y},{ }_{p 1} I_{x},{ }_{p 1} I_{y},{ }_{s 2} I_{x, s_{2}} I_{y}$, ${ }_{p 2} I_{x}$, and ${ }_{p 2} I_{y}$. Note that the eight measured intensities overdetermine the five fitted angular parameters.

The above setup resolves angles within one quarter of a hemisphere (e.g., $0<\theta<90^{\circ}$ and $0<\phi<$ $90^{\circ}$ ). This ambiguity can be reduced by incorporating additional polarizations into each beam intermediate $\left(\right.$ at $45^{\circ}$ ) between the vertical and horizontal orientations. The additional polarizations in both beams (for a total of 16 measured intensities; see Fig. 3, top left) increase the angular range to a full hemisphere (e.g., $0<\theta<180^{\circ}, 0<\phi<180^{\circ}$ ). This increase in angular range requires additional polarization measurements, totaling 16 traces, which doubles the cycle time and reduces the time resolution for the duration of a given recording interval.

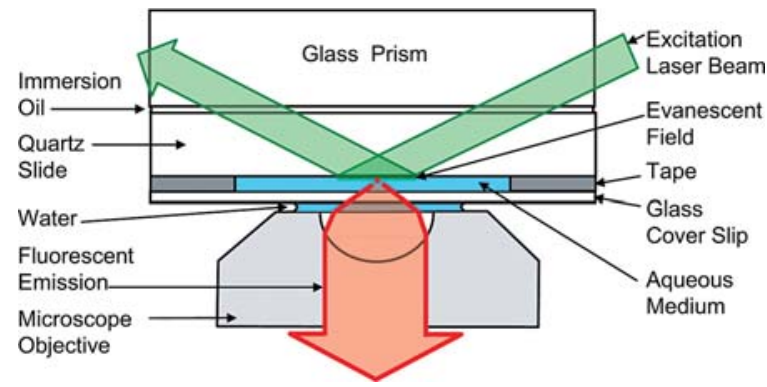

FIGURE 4. A cross section of the polTIRFM setup at the sample, showing the glass coupling prism, the flow cell, and the objective lens of the microscope (not drawn to scale). The autofluorescence from the glass prism is generated outside the field of view and thus does not contribute significantly to the background intensity. 
The processive motility assay and the twirling assay can be used for determining the three-dimensional orientation and wobble of a fluorophore. In the processive motility assay, CaM is labeled with BR and is exchanged onto the myosin $\mathrm{V}$ lever arm. As the myosin moves along a stationary actin filament, the orientation ( $\beta$ in particular) of the fluorophore changes when the bound head steps between the leading and trailing positions. In the twirling assay, actin filaments that are sparsely labeled with rhodamine translocate on a myosin-coated slide. Approximately one-third to one half of the gliding filaments rotate about their axis (i.e., the $\alpha$ angle), thereby imposing a helical path on the fluorescent probe. Such filaments are said to twirl, and when driven by myosin $\mathrm{V}$, they have a consistent lefthanded pitch that averages $\sim 1 \mu \mathrm{m}$.

The protocols Fluorescent Labeling of Calmodulin with Bifunctional Rhodamine (Beausang et al. 2012a) and Fluorescent Labeling of Myosin V for Polarized Total Internal Reflection Fluorescence Microscopy (polTIRFM) Motility Assays (Beausang et al. 2012b) are required for labeling the myosin $\mathrm{V}$ lever arm used in the processive motility assay. CaM is labeled with BR, and BR-CaM is exchanged at low stoichiometry for the endogenous CaM in the myosin. The protocol Preparation of Filamentous Actin for Polarized Total Internal Reflection Fluorescence Microscopy (polTIRFM) Motility Assays (Beausang et al. 2012c) describes various methods for labeling actin that are used in both motility assays. When possible, all solutions should be prepared using $0.2-\mu \mathrm{m}$ filtered, deionized water in a clean hood while wearing gloves to prevent sample contamination. The techniques for myosin $\mathrm{V}$ should be generally applicable to other single-molecule experiments where angular changes have an important mechanistic role in their biological function.

This work was funded by National Institutes of Health grants AR26846 and AR51174 to the Pennsylvania Muscle Institute and by the National Science Foundation NSEC Nano/Bio Interface Center (NBIC) DMR 04-25780. J.F.B. is supported by an NSF IGERT fellowship through the NBIC DGE 0221664. We thank Drs. Philip C. Nelson and Jody A. Dantzig-Brody for review and useful comments on the manuscript.

\section{REFERENCES}

Adams SR, Campbell RE, Gross LA, Martin BR, Walkup GK, Yao Y, Llopis J, Tsien RY. 2002. New biarsenical ligands and tetracysteine motifs for protein labeling in vitro and in vivo: Synthesis and biological applications. J Am Chem Soc 124: 6063-6076.

Ali MY, Uemura S, Adachi K, Itoh H, Kinosita K Jr, Ishiwata S. 2002. Myosin $\mathrm{V}$ is a left-handed spiral motor on the right-handed actin helix. Nat Struct Biol 9: 464-467.

Asenjo AB, Weinberg Y, Sosa H. 2006. Nucleotide binding and hydrolysis induces a disorder-order transition in the kinesin neck-linker region. Nat Struct Mol Biol 13: 648-654.

Axelrod D. 1989. Fluorescence polarization microscopy. Methods Cell Biol 30: $333-352$.

Bartko AP, Xu K, Dickson RM. 2002. Three-dimensional single molecule rotational diffusion in glassy state polymer films. Phys Rev Lett 89: 026101.1-026101.4.

Beausang JF, Sun Y, Quinlan ME, Forkey JN, Goldman YE. 2012a. Fluorescent labeling of calmodulin with bifunctional rhodamine. Cold Spring Harb Protoc doi: 10.1101/pdb.prot069351.

Beausang JF, Sun Y, Quinlan ME, Forkey JN, Goldman YE. 2012b. Fluorescent labeling of myosin $\mathrm{V}$ for polarized total internal reflection fluorescence microscopy (polTIRFM) motility assays. Cold Spring Harb Protoc doi: $10.1101 /$ pdb.prot069369.

Beausang JF, Sun Y, Quinlan ME, Forkey JN, Goldman YE. 2012c. Preparation of filamentous actin for polarized total internal reflection fluorescence microscopy (polTIRFM) motility assays. Cold Spring Harb Protoc doi: 10.1101/pdb.prot069377.

Betzig E, Patterson GH, Sougrat R, Lindwasser OW, Olenych S, Bonifacino JS, Davidson MW, Lippincott-Schwartz J, Hess HF. 2006. Imaging intracellular fluorescent proteins at nanometer resolution. Science 313: 1642-1645.

Brack AS, Brandmeier BD, Ferguson RE, Criddle S, Dale RE, Irving M. 2004. Bifunctional rhodamine probes of myosin regulatory light chain orientation in relaxed skeletal muscle fibers. Biophys J 86: 2329-2341.

Burgess WH, Jemiolo DK, Kretsinger RH. 1980. Interaction of calcium and calmodulin in the presence of sodium dodecyl sulfate. Biochim Biophys Acta 623: 257-270.

Chen RF, Bowman RL. 1965. Fluorescence polarization: Measurement with ultraviolet-polarizing filters in a spectrophotofluorometer. Science 147: 729-732.

Chiu W, Baker ML, Almo SC. 2006. Structural biology of cellular machines. Trends Cell Biol 16: 144-150.

Corrie JET, Craik JS, Munasinghe VRN. 1998. A homo-bifunctional rhodamine for labeling proteins with defined orientations of a fluorophore. Bioconjug Chem 9: 160-167.

Corrie JET, Brandmeier BD, Ferguson RE, Trentham DR, Kendrick-Jones J, Hopkins SC, van der Heide UA, Goldman YE, Sabido-David C, Dale RE, et al. 1999. Dynamic measurement of myosin light-chain-domain tilt and twist in muscle contraction. Nature 400: 425-430. 
Costello MJ. 2006. Cryo-electron microscopy of biological samples. Ultrastruct Pathol 30: 361-371.

Enderlein J, Toprak E, Selvin PR. 2005. Polarization effect on position accuracy of fluorophore localization. Opt Express 14: 8111-8120.

Ferguson RE, Sun YB, Mercier P, Brack AS, Sykes BD, Corrie JET, Trentham DR, Irving M. 2003. In situ orientations of protein domains: Troponin C in skeletal muscle fibers. Mol Cell 11: 865-874.

Forkey JN, Quinlan ME, Goldman YE. 2000. Protein structural dynamics by single-molecule fluorescence polarization. Prog Biophys Mol Biol 74: $1-35$.

Forkey JN, Quinlan ME, Shaw MA, Corrie JET, Goldman YE. 2003. Threedimensional structural dynamics of myosin $\mathrm{V}$ by single-molecule fluorescence polarization. Nature 422: 399-404.

Forkey JN, Quinlan ME, Goldman YE. 2005. Measurement of single macromolecule orientation by total internal reflection fluorescence polarization microscopy. Biophys J 89: 1261-1271.

Geeves MA, Holmes KC. 2005. The molecular mechanism of muscle contraction. Adv Protein Chem 71: 161-193.

Goldman YE. 1998. Wag the tail: Structural dynamics of actomyosin. Cell 93: $1-4$.

Gore J, Bryant Z, Stone MD, Nöllmann M, Cozzarelli NR, Bustamante C. 2006. Mechanochemical analysis of DNA gyrase using rotor bead tracking. Nature 439: 100-104.

Gutfreund H. 1995. Kinetics for the life sciences: Receptors, transmitters and catalysts Cambridge University Press, Cambridge.

Ha T, Glass J, Enderle T, Chemla DS, Weiss S. 1998. Hindered rotational diffusion and rotational jumps of single molecules. Phys Rev Lett 80: 2093-2096.

Hell SW, Dyba M, Jakobs S. 2004. Concepts for nanoscale resolution in fluorescence microscopy. Curr Opin Neurobiol 14: 599-609.

Hopkins SC, Sabido-David C, van der Heide UA, Ferguson RE, Brandmeier BD, Dale RE, Kendrick-Jones J, Corrie JET, Trentham DR, Irving M, et al. 2002. Orientation changes of the myosin light chain domain during filament sliding in active and rigor muscle. J Mol Biol 318: 1275-1291.

Houdusse A, Silver M, Cohen C. 1996. A model of $\mathrm{Ca}^{2+}$-free calmodulin binding to unconventional myosins reveals how calmodulin acts as a regulatory switch. Structure 4: 1475-1490.

Hua W, Chung J, Gelles J. 2002 . Distinguishing inchworm and hand-overhand processive kinesin movement by neck rotation measurements. Science 295: 844-848.

Igumenova TI, Frederick KK, Wand AJ. 2006. Characterization of the fast dynamics of protein amino acid side chains using NMR relaxation in solution. Chem Rev 106: 1672-1699.

Irving M, St Claire Allen T, Sabido-David C, Craik JS, Brandmeier B, Kendrick-Jones J, Corrie JET, Trentham DR, Goldman YE. 1995. Tilting of the light-chain region of myosin during step length changes and active force generation in skeletal muscle. Nature 375: 688-691.

Kinosita K Jr. 1999. Real time imaging of rotating molecular machines. FASEB J 2: S201-S208.

Kinosita K Jr, Adachi K, Itoh H. 2004. Rotation of F1-ATPase: How an ATPdriven molecular machine may work. Annu Rev Biophys Biomol Struct 33: $245-268$

Kosolapov A, Deutsch C. 2003. Folding of the voltage-gated $\mathrm{K}^{+}$channel T1 recognition domain. J Biol Chem 278: 4305-4313.

Kreiter M, Prummer M, Hecht B, Wild UP. 2002. Orientation dependence of fluorescence lifetimes near an interface. J Chem Phys 117: 9430-9433.

La Porta A, Wang MD. 2004. Optical torque wrench: Angular trapping, rotation, and torque detection of quartz microparticles. Phys Rev Lett 92: 190801.

Lakowicz J.R. 2006. Principles of fluorescence spectroscopy, 3rd ed. Springer, New York.

Lieb MA, Zavislan JM, Novotny L. 2004. Single-molecule orientations determined by direct emission pattern imaging. J Opt Soc Am B 21: $1210-1215$.

Marks KM, Nolan GP. 2006. Chemical labeling strategies for cell biology. Nat Methods 3: 591-596.

Mercier P, Ferguson RE, Irving M, Corrie JET, Trentham DR, Sykes BD. 2003. NMR structure of a bifunctional rhodamine labeled N-domain of troponin C complexed with the regulatory "switch" peptide from troponin I: Implications for in situ fluorescence studies in muscle fibers. Biochemistry 42: 4333-4348.

Min W, English BP, Luo G, Cherayil BJ, Kou SC, Xie XS. 2005. Fluctuating enzymes: Lessons from single-molecule studies. Acc Chem Res 38: 923-931.

Otterbein LR, Graceffa P, Dominguez R. 2001. The crystal structure of uncomplexed actin in the ADP state. Science 293: 708-711.

Penzkofer A, Wiedmann J. 1980. Orientation of transition dipole moments of rhodamine $6 \mathrm{G}$ determined by excited state absorption. Optics Commun 35: 81-86.

Pollard TD, Earnshaw WC. 2002. Cell biology. Saunders, Philadelphia.

Press WH, Teukosky SA, Vetterling WT, Flannery BP. 1992. Numerical recipes in $C$, 2nd ed. Cambridge University Press, Cambridge.

Quinlan ME, Forkey JN, Goldman YE. 2005. Orientation of the myosin light chain region by single molecule total internal reflection fluorescence polarization microscopy. Biophys J 89: 1132-1142.

Rasnik I, McKinney SA, Ha T. 2006. Nonblinking and long-lasting singlemolecule fluorescence imaging. Nat Methods 3: 891-893.

Rosenberg SA, Quinlan ME, Forkey JN, Goldman YE. 2005. Rotational motions of macro-molecules by single-molecule fluorescence microscopy. Acc Chem Res 38: 583-593.

Robinson JM, Kosolapov A, Deutsch C. 2006. Tertiary and quaternary structure formation of voltage-gated potassium channels. Methods Mol Biol 337: 41-52.

Rust MJ, Bates M, Zhuang X. 2006. Sub-diffraction-limit imaging by stochastic optical reconstruction microscopy (STORM). Nat Methods 3: 793-795.

Sale K, Sár C, Sharp KA, Hideg K, Fajer PG. 2002. Structural determination of spin label immobilization and orientation: A Monte Carlo minimization approach. J Magn Reson 156: 104-112.

Seidel R, Dekker C. 2007. Single-molecule studies of nucleic acid motors. Curr Opin Struct Biol 17: 80-86.

Senior AE, Nadanaciva S, Weber J. 2002. The molecular mechanism of ATP synthesis by F1F0-ATP synthase. Biochim Biophys Acta 1553: 188-211.

Shaevitz JW, Block SM, Schnitzer MJ. 2005. Statistical kinetics of macromolecular dynamics. Biophys J 89: 2277-2285.

Shiroguchi K, Kinosita K Jr. 2007. Myosin V walks by lever action and Brownian motion. Science 316: 1208-1212.

Sick B, Hecht B, Novotny L. 2000. Orientational imaging of single molecules by annular illumination. Phys Rev Lett 85: 4482-4485.

Sosa H, Peterman EJG, Moerner WE, Goldstein LSB. 2001. ADP-induced rocking of the kinesin motor domain revealed by single-molecule fluorescence polarization microscopy. Nat Struct Biol 8: 540-544.

Strick T, Allemand JF, Croquette V, Bensimon D. 2000. Twisting and stretching single DNA molecules. Prog Biophys Mol Biol 74: 115-140.

Sun YB, Brandmeier B, Irving M. 2006 . Structural changes in troponin in response to $\mathrm{Ca}^{2+}$ and myosin binding to thin filaments during activation of skeletal muscle. Proc Natl Acad Sci 103: 17771-17776.

Syed S, Snyder GE, Franzini-Armstrong C, Selvin PR, Goldman YE. 2006. Adaptability of myosin $\mathrm{V}$ studied by simultaneous detection of position and orientation. EMBO J 25: 1795-1803.

Toprak E, Enderlein J, Syed S, McKinney SA, Petschek RG, Ha T, Goldman YE, Selvin PR. 2006. Defocused orientation and position imaging (DOPI) of myosin V. Proc Natl Acad Sci 103: 6495-6499.

Toyoshima C, Inesi G. 2004. Structural basis of ion pumping by $\mathrm{Ca}^{2+}$ ATPase of the sarcoplasmic reticulum. Annu Rev Biochem 73: 269-292.

van der Heide UA, Orbons B, Gerrtsen HC, Levine YK. 1992. The orientation of transition moments of dye molecules used in fluorescence studies of muscle systems. Eur Biophys J 21: 263-272.

Veigel C, Wang F, Bartoo ML, Sellers JR, Molloy JE. 2002. The gated gait of the processive molecular motor, myosin V. Nat Cell Biol 4: 59-65.

Waggoner A. 2006. Fluorescent labels for proteomics and genomics. Curr Opin Chem Biol 10: 62-66.

Wilson T, Massoumian F, Jusvskaitas R. 2003. Generation and focusing of radially polarized electric fields. Opt Engr 42: 3088-3089.

Yildiz A, Forkey JN, McKinney SA, Ha T, Goldman YE, Selvin PR. 2003. Myosin V walks hand-over-hand: Single fluorophore imaging with 1.5-nm localization. Science 300: 2061-2065. 


\section{Orientation and Rotational Motions of Single Molecules by Polarized Total Internal Reflection Fluorescence Microscopy (polTIRFM)}

John F. Beausang, Yujie Sun, Margot E. Quinlan, Joseph N. Forkey and Yale E. Goldman

Cold Spring Harb Protoc; doi: 10.1101/pdb.top069344

\begin{tabular}{|c|c|}
\hline $\begin{array}{r}\text { Email Alerting } \\
\text { Service }\end{array}$ & Receive free email alerts when new articles cite this article - click here. \\
\hline $\begin{array}{l}\text { Subject } \\
\text { Categories }\end{array}$ & $\begin{array}{l}\text { Browse articles on similar topics from Cold Spring Harbor Protocols. } \\
\text { Fluorescent Proteins ( } 259 \text { articles) } \\
\text { Light Microscopy ( } 61 \text { articles) } \\
\text { Liquid Chromatography ( } 51 \text { articles) } \\
\text { Protein: Protein Interactions ( } 86 \text { articles) } \\
\text { Visualization of Proteins ( } 107 \text { articles) }\end{array}$ \\
\hline
\end{tabular}

